\title{
Investigation of acute stroke: what is the most effective strategy?
}

\author{
D.W. Dunbabin and P.A.G. Sandercock
}

Department of Clinical Neurosciences, Western General Hospital, Edinburgh EH4 2XU, UK

\begin{abstract}
Summary: Techniques of investigation of acute stroke syndromes have progressed rapidly in recent years, outpacing developments in effective stroke treatment. The clinician is thus faced with a variety of tests, each with different cost implications and each altering management to a greater or lesser extent. This review will concentrate on the basic tests which should be performed for all strokes (full blood count, ESR, biochemical screen, blood glucose, cholesterol, syphilis serology, chest X-ray and electrocardiogram). Additional tests may be required in selected cases: CT scan to diagnose 'non-stroke' lesions, to exclude cerebral haemorrhage if anti-haemostatic therapy is planned, and to detect strokes which may require emergency intervention (such as cerebellar stroke with hydrocephalus); echocardiography to detect cardiac sources of emboli; and in a few cases lumbar puncture and specialized haematological tests. Other tests, which are currently research tools, may be suitable for widespread use in the future including NMR, SPECT and PET scanning.
\end{abstract}

\section{Introduction}

\section{The problem of stroke}

In the next year about 100,000 people in the United Kingdom will have a definite first stroke and many others will have symptoms suggestive of stroke. Of the definite strokes about 20,000 will die and about 30,000 survivors will be seriously disabled. ${ }^{1}$ After recovery from stroke the survivors will be at risk of recurrence and other vascular events such as myocardial infarction and vascular death. The economic burden of stroke is large, approximately $5 \%$ of the total National Health Service budget. ${ }^{2}$ There will be additional direct costs to the Social Services and indirect costs to the community from the lost income of unpaid carers.

About $85 \%$ of strokes are due to cerebral infarction (caused by occlusion of extracranial or intracranial cerebral vessels), $10 \%$ are due to primary intracerebral haemorrhage and $5 \%$ are due to subarachnoid haemorrhage. ${ }^{3}$ Stroke is a heterogeneous disease with a wide variety of clinical presentations, each associated with differing prognosis. The clinical management of patients in the acute phase of stroke remains controversial. Imaging and cerebral function techniques have advanced enormously, yet the most appropriate scheme of investigation and the most

Correspondence: P.A.G. Sandercock, D.M., M.R.C.P. Received: 13 September 1990 effective treatment of a patient with suspected acute stroke is not clear, a contrast to the investigation and management of other occlusive vascular diseases such as myocardial infarction. This review will concentrate on strategies for the investigation of all forms of suspected stroke with the exception of the clinically distinct syndrome of subarachnoid haemorrhage, which has recently been reviewed very well by Vermeulen. ${ }^{4}$ We will also briefly review the general principles of the assessment of diagnostic tests and then the value of specific tests in the assessment of suspected stroke.

\section{Why investigate a stroke?}

'Errors in judgment must occur in the practice of an art which consists largely in balancing probabilities.' Sir William Osler.

The reasons for investigating an individual patient presenting with suspected acute stroke are varied. Decision analysis with formal modelling may provide a better way of selecting appropriate tests, but is still a relatively crude indicator of the most cost-effective form of investigation. Algorithm flowcharts can provide a rigid protocol to follow, but may need to be altered as technology advances, or to suit different institutions (with differing patterns of disease presenting and differing investigational facilities), or to suit the views of different 
physicians (depending on their view of what constitutes the best treatment). Nonetheless, a systematic approach should ensure that the investigation of patients with stroke becomes more rational, more cost-effective and less risky.

\section{General aims of investigation}

(a) Confirm the diagnosis of stroke The main differential diagnoses for stroke are listed in Table I. The majority of these can be excluded by careful attention to establishing the history. If there is a clear history of sudden onset, non-stroke lesions are very unlikely. If there is doubt about speed of onset or progression or if the patient is confused, dysphasic or comatose, a telephone call (cost, a few pence) to talk to a witness or member of the close family may be the most cost-effective investigation and is certainly easier to obtain than a computed tomographic (CT) scan (cost $£ 50-80$ ). Surveys of admissions with suspected stroke to stroke units and neurological wards reveal a 5-13\% incidence of 'non-stroke' lesions, largely unrecognized seizures or confusional states. ${ }^{5-7}$ In a communitybased sample of patients the proportion of 'nonstroke' lesions will be lower $1.5 \%,{ }^{3}$ reflecting admission bias; a patient with an atypical clinical picture associated with a 'non-stroke' lesion is more likely to be admitted to hospital.

(b) Clarify the pathology The distinction between cerebral infarction and primary intracerebral haemorrhage may be important if anti-haemostatic treatment is contemplated. A scoring system based on clinical features enables the calculation of a probability ratio for cerebral haemorrhage, ${ }^{7}$ but this may incorrectly classify some small deep haemorrhages as infarcts. The mechanism of infarction may be identified; for example differentiating between arterial or venous occlusion

Table I Nonvascular lesions which can present with the clinical syndrome of stroke

intracranial tumour (primary or metastatic)

subdural haematoma

post-ictal paralysis (Todd's paresis)

intracranial infection (encephalitis, meningitis or abscess)

hypoglycaemia

hyperglycaemia (especially nonketotic)

electrolyte disturbance (low serum sodium, phosphate etc.)

multiple sclerosis (rare)

occult head injury

peripheral nerve lesion

hysteria

migraine and the type of occlusion may sometimes be determined (whether there is in situ thrombosis or $z$ embolism from the heart or from atheromatous $\mathbb{\Phi}$ arterial plaques).

(c) To discover the cause There are many $\underset{\vec{P}}{\vec{P}}$ potential causes of cerebral infarction, but the most ${ }^{\circ}$ common is arterial occlusion, related to embolic $\underline{\underline{B}}$ and thrombotic consequences of atherosclerotic $\frac{\omega}{\square}$ disease of arteries to the brain. A small proportion $\stackrel{\odot}{\circ}$ of strokes (Table II) are related to non-atheromatous, non-embolic causes and are important $\vec{\circ}$ because they are often amenable to treatment.

(d) As a guide to prognosis Some techniques provide information which predicts prognosis, but 8 clinical data such as age, level of consciousness, the 3 . severity of neurological defect, perceptual defect $\sigma^{2}$ and the development of incontinence are powerful predictors of outcome; $;^{8-12}$ studies are now needed or to see whether new imaging or laboratory tech- i nology, if added to current clinical prognostic 0 scoring systems, improves prognostic accuracy.

\section{(e) As a guide to treatment}

(i) Acute phase of stroke. For the majority of strokes there is no currently available, widef $\vec{\oplus}$ practicable treatment which has been demor strated to improve survival or reduce the function handicap after stroke. Many clinicians feel thăt there is little point in extensive investigation of $\bar{\partial}$ patients soon after stroke onset if no effective $\stackrel{\otimes}{0}$ treatment is available. This rather nihilistic view of $\stackrel{\AA}{\propto}$ stroke ignores the small proportion of patients who $\overrightarrow{\vec{P}}$ have a non-stroke lesion or a treatable type of $\frac{0}{3}$ stroke (Tables I and II). If effective treatments for stroke become available it will be important to identify those who may benefit. For example, if $-\overline{0}$ early thrombolysis and arterial recanalization is 3 . shown to improve outcome it will become impor- $\dot{\sigma}$ tant to identify patients with a cerebral infarction, persisting occlusion of a major cerebral artery, and $\delta$ with potentially viable ischaemic tissue before treatment is started.

(ii) Long-term secondary prevention. If long-term treatment with antiplatelet or anticoagulants is planned, or if the patient is at high risk of deep vein thrombosis (and may require anticoagulation) it is $\odot$ necessary to exclude primary intracerebral haem- $N$ orrhage before starting treatment.

(f) To make or to save money Investigating patients purely for profit is not a practice which $\frac{}{\Phi}$ most clinicians would readily admit to, but may be $\stackrel{\oplus}{\rightarrow}$ a powerful determinant of medical practice in some 0 parts of the world. More relevant to the National $\overline{0}$ Health Service, an investigation may, by avoiding a $\stackrel{\otimes}{\odot}$ disabling mis-diagnosis or by identifying appropri- $\bar{\Phi}$ 
Table II Causes of stroke which need specific treatment or need to be identified for other reasons

CEREBRAL INFARCTION

infective arteritis - local sepsis, syphilis

inflammatory arteritis - giant-cell, systemic lupus erythematosus, Moya-Moya, fibromuscular dysplasia, polyarteritis nodosa, granulomatous angiitis

cardiogenic embolism - rheumatic heart disease, myxomatous mitral valve disease, endocarditis, mural thrombus, paradoxical embolus, atrial myxoma, cardiac arrhythmia

arterial dissection - traumatic, spontaneous, Marfan's, homocystinuria

coagulopathy - sex steroids, protein C \& S deficiency, hyperviscosity syndromes, polycythaemia, anticardiolipin antibodies, sickle-cell disease

drugs - cocaine, amphetamines

systemic hypotension - antihypertensive drugs, surgery, blood loss, bradycardia

decompression sickness

gas or fat embolus

cerebellar infarction with obstructive hydrocephalus

cerebral venous thrombosis

PRIMARY INTRACEREBRAL HAEMORRHAGE

coagulopathy - iatrogenic anticoagulant overdose,

disseminated intravascular coagulation, haemophilia congenital vascular malformations

impaired platelet number or function - leukaemia,

idiopathic thrombocytopaenic purpura, aplasia

drugs - cocaine

amyloid degeneration

cerebellar haemorrhage with obstructive hydrocephalus cerebral venous thrombosis

ate treatment, reduce disability and hence reduce the length of hospital stay.

\section{Evaluation of an investigative test}

The precision with which an individual test can identify the presence or absence of disease can be expressed in a number of measures which are assessed in a formal study comparing the tests performance with a 'gold standard' test. For example, Doppler ultrasonography of the carotid arteries can be assessed by comparison with the 'gold standard' of intra-arterial angiography to determine sensitivity (the probability of detecting disease when it is present) and specificity (the probability of excluding disease when it is absent) for the detection of carotid stenosis. The positive predictive value of a test is the proportion of patients with a positive test result who have the disease and the negative predictive value is the proportion of patients with negative test results who do not have the disease. Sensitivity and specificity do not change with alterations in the prevalence of disease in the population under test. However, the positive predictive value is greatly influenced by the prevalence of disease in the population assessed and this must be taken into account when interpreting any test result. Most tests perform best when the prevalence of disease (pre-test probability) is about $50 \%$; a positive or negative test result can then contribute significantly to the diagnosis by substantially increasing or decreasing the post-test probability of disease. For example, when the pre-test probability is low (say $10 \%$ ) or high (say $90 \%$ ), then the result of the diagnostic test will change those probabilities only slightly and add little to the clinical diagnosis. ${ }^{13}$ An illustration of the ability of Doppler ultrasound studies to detect significant carotid stenosis in different types of patient with differing pre-test probabilities of disease is given in Table III. Of course other factors must also be borne in mind when assessing how good a particular test is: the cost, the risk of side effects, the expertise of local diagnostic services (measures of sensitivity and specificity are usually derived from centres of excellence) and the extent to which the test result can usefully influence management.

Rather than outline a 'stroke workup' or flowchart to be applied rigidly to all patients, we will review each of the simple tests which are widely available at present and propose what we consider a cost-effective basic minimum set. For the remainder, we will discuss those investigations which may be appropriate in selected cases and then the procedures which are research tools at present but may have relevance in the future.

\section{Basic investigations applicable to every patient}

These are listed in Table IV. They have remained largely unchanged since 1950 when the authors of Harrison's Principles of Internal Medicine suggested urinalysis, determination of blood nitrogen and sugar levels, skull radiography and cerebrospinal fluid examination. ${ }^{14}$ The tests cost little and are in widespread use; it is perhaps not surprising that there have been no formal studies to assess the utility of each test. Many non-hospitalized patients still do not have these investigations performed, but perhaps should do. The aim of these tests is to supplement the clinical search for treatable conditions.

Full blood count may point to rare causes of stroke such as blood dyscrasia, polycythaemia, thrombocytopaenia, thrombocytosis or infection.

$E S R$-its role is to screen for vasculitis, bacterial endocarditis or hyperviscosity as the cause of stroke.

Biochemical screen may provide evidence that the neurological deficit is due to metabolic disturbance 
Table III The ability of duplex ultrasonography of the carotids to detect moderate to severe carotid stenosis (greater than $50 \%$ decrease in diameter) in patients with differing pre-test probabilities of disease assuming a sensitivity and specificity of approximately $95 \%$ against the gold standard of carotid angiography

\begin{tabular}{|c|c|c|}
\hline History & $\begin{array}{c}\text { Pre-test } \\
\text { probability }\end{array}$ & $\begin{array}{l}\text { Post-test } \\
\text { probability }\end{array}$ \\
\hline $\begin{array}{l}40 \text { year old woman with visual loss possibly } \\
\text { migrainous, possibly vascular }\end{array}$ & $5 \%$ & $\begin{array}{l}+ \text { ve test }=>50 \% \\
- \text { ve test }=>0.3 \%\end{array}$ \\
\hline $\begin{array}{l}45 \text { year old man with visual loss hypertension } \\
\text { and ?bruit }\end{array}$ & $50 \%$ & $\begin{array}{l}+ \text { ve test }=>95 \% \\
- \text { ve test }=>5 \%\end{array}$ \\
\hline $\begin{array}{l}68 \text { year old man with definite carotid transient } \\
\text { ischaemic attacks, angina, peripheral vascular } \\
\text { disease and loud carotid bruits }\end{array}$ & $95 \%$ & $\begin{array}{l}+ \text { ve test }=>99.7 \% \dagger \\
- \text { ve test }=>50 \%\end{array}$ \\
\hline
\end{tabular}

*Test may be useful here to exclude presence of disease but will generate 5 false-positive results in every 100 patients screened (i.e. test positive but stenosis not present). $† A$ positive test here adds little to the clinical suspicion of disease.

Table IV Basic investigations applicable to every patient with suspected acute stroke

Full blood count and ESR

Urea and electrolytes, calcium

Blood glucose

Cholesterol

Serological tests for syphilis

Chest radiography

Electrocardiograph

or electrolyte imbalance rather than a vascular lesion of the brain.

Blood glucose Hypoglycaemia and non-ketotic hyperglycaemia can both be associated with striking focal neurological deficits which mimic stroke, yet disappear completely when plasma glucose levels are returned to normal. This test also helps diagnose occult diabetes mellitus and provides a prognostic indicator (elevated blood glucose after stroke, even in non-diabetics, is an indicator of adverse outcome and increased infarct size, although whether this reflects a cause-and-effect relationship is unclear). ${ }^{15}$

Serum cholesterol-if taken within 12-24 hours of the acute event it represents a reliable measure of the pre-stroke levels. This may predict occurrence of further vascular events, and may represent a risk factor for modification in secondary prevention. If this test is not performed acutely then it should be delayed for at least 6 weeks because of changes in cholesterol level induced by the stress of the acute stroke.

Syphilis serology Meningovascular syphilis is a rare cause of stroke (a frequency of 1 in 500 strokes in a recent community survey ${ }^{3}$ ), but one which is potentially treatable, so screening using a non- treponemal test such as the VDRL is probably cost-effective. $^{16}$

Chest radiography provides, with the clinical history and electrocardiogram (ECG), evidence of potential cardioembolic lesions. In addition heart size can indicate prolonged hypertension. Less commonly, unsuspected malignancy may be found, and the chest X-ray may enable early diagnosis of complications such as pneumonia and pulmona embolism to be made.

Electrocardiography In occasional patients with stroke associated with complete heart block it is diagnostic. Otherwise, the ECG provides confirmatory evidence of underlying cardiovascular disease such as ischaemic heart disease, arrhythmia or valvular lesions. Prospective ECG, cardiac enzyme and autopsy studies have suggested that asymptomatic myocardial necrosis occurs in many patients during the acute phase of stroke, although the effect on outcome is unclear. ${ }^{17}$

\section{Additional investigations in special circumstances}

In general, use of these tests is guided by clinical suspicion or an abnormal result on one of the basic tests.

\section{(A) Haematological investigations}

Serum protein levels, plasma viscosity and electrophoresis may be of use if hyperviscosity is suspected, usually the ESR is high in these patients.

Autoantibody screen in younger patients or in patients with suspected autoimmune disease or vasculitis. A prolonged activated partial thromboplastin time (APTT) or nonspecific syphilis 
serology may suggest the presence of anticardiolipin antibodies. Even in the absence of clinical signs of systemic lupus erythematosus the presence of these antibodies in high titre has been associated with idiopathic stroke in young patients, and may identify patients at particularly high risk of recurrent vascular events. ${ }^{18}$

Sickle test in appropriate racial groups (Negroes).

Haemostatic profile in patients with haemorrhagic stroke not due to hypertension, aneurysm or arteriovenous malformation, tests of haemostasis including prothrombin time, activated partial thromboplastic time, thrombin time, bleeding time or fibrin degradation products may be required. In patients with unexplained ischaemic stroke (especially young patients or in those with a strong past or family history of premature arterial or venous occlusive disease), measurement of protein $\mathrm{S}$ and $\mathrm{C}$ levels, antithrombin III levels, platelet aggregation or fibrinolytic activity may be helpful. Thrombotic disorders due to genetic abnormalities in the clotting system are relatively rare. The acquired thrombotic tendency associated with 'lupus anticoagulant' (anticardiolipin antibodies) is an increasingly recognized cause of unexplained ischaemic stroke, ${ }^{18}$ and initial screening should be requested in consultation with a haematologist. Factor VIII and fibrinogen levels are associated with an increased risk of stroke and may predict outcome and the risk of recurrence. ${ }^{19}$

\section{(B) Urine tests}

Screening for homocystinuria with methionine loading, or tests for porphyria or metanephrines to detect phaeochromocytoma may be sometimes indicated.

\section{(C) Lumbar puncture}

This investigation may occasionally be helpful by excluding lesions mimicking stroke, such as meningitis, encephalitis, or to confirm subarachnoid haemorrhage. In cases of suspected subarachnoid haemorrhage computed tomography of the brain is the preferred initial investigation. ${ }^{4}$ The ability of cerebrospinal fluid (CSF) examination to discriminate between primary cerebral haemorrhage and cerebral infarction has been examined in two prospective studies. In one, a CSF protein, measured a mean of 2.5 days after stroke onset, greater than $1 \mathrm{~g} / 1$ had a sensitivity of $89 \%$ and a specificity of $92 \%$ for haemorrhagic stroke whilst visible xanthochromia showed a sensitivity of $70 \%$ and a specificity of $95 \%$ where the gold standard was CT or autopsy diagnoses. ${ }^{20}$ In centres with no CT scanning facilities, lumbar puncture (LP) and CSF examination are still used to exclude intracerebral haemorrhage; however, LP is clearly inferior to CT scanning particularly in the diagnosis of small deep haemorrhages and does not exclude haemorrhages reliably enough to permit anticoagulant therapy. ${ }^{21}$ The danger of a spinal haematoma complicating early anticoagulation after lumbar puncture is significant, seven cases of iatrogenic paraplegia were reported in a series of 342 patients. ${ }^{21}$ LP should not be performed if there is any evidence of raised intracranial pressure.

\section{(C) Computed tomography of the brain}

CT scanning is very informative in stroke for several reasons. In the few patients for whom a clear history of a sudden onset of focal neurological deficit cannot be obtained, it differentiates 'stroke' from 'non-stroke' lesions. It can discriminate between haemorrhage and infarction reliably at an early stage, and may later clarify the site and type of infarct and the likely underlying vascular pathology, ${ }^{22}$ with good interobserver agreement. ${ }^{23}$ CT scanning would thus appear to be desirable for all cases of stroke, but is it necessary? Sandercock et al. calculated that the cost of performing CT scans on every patient who presented with a first stroke in England and Wales would be at least $£ 1.35$ million per year (1984 prices), ${ }^{24}$ without the additional expense of providing CT scanners to the $49 \%$ of health districts and boards which do not currently have such equipment. ${ }^{25}$

(1) Differentiating 'stroke' from 'nonstroke' The incidence of non-stroke lesions detected by CT scanning in patients with suspected stroke ranges from $1.5-16 \% .{ }^{24,26-30}$ In about two thirds of patients, the detection of a 'non-stroke' lesion results in major changes to management. ${ }^{24,26}$ The frequency of unexpected non-stroke lesions in a population of patients clinically suspected of stroke can be reduced by careful elucidation of the history, particularly the speed of onset of neurological deficit. The corollary is that in cases where no clear history is available from the patient or a reliable witness, a CT scan may be needed to confirm the clinical diagnosis of stroke.

(2) Excluding haemorrhagic stroke CT scanning represents the best method of excluding haemorrhage (CSF examination has a sensitivity of only $60-70 \%$ against the gold standard of CT scanning). ${ }^{31}$ During the first 24-48 hours after onset of stroke a primary intracerebral haemorrhage will be clearly visible as an area of marked hyperdensity provided the haemorrhage is greater than about $5 \mathrm{~mm}$ in diameter; this is probably the best time to detect small haemorrhages. The den- 
sity of haemorrhages declines with time, so that by 14-21 days the haematoma can be isodense with brain and thus invisible, or hypodense and indistinguishable from a small lacunar infarct. ${ }^{32}$ Infarcts on early CT scan may also undergo haemorrhagic transformation at a later date in approximately $20 \%$ of acute cerebral infarctions which were scanned within 6 hours of onset in one series. ${ }^{34}$ All haemorrhagic transformations occurred less than one month after onset of stroke. To reliably discriminate between haemorrhagic and ischaemic stroke CT scanning less than 3 weeks after onset is mandatory.

In suspected cardioembolic stroke or stroke which appears to be progressing, some clinicians use anticoagulant therapy, although its efficacy in altering the natural history of acute stroke is not yet clear. The role of antiplatelet therapy in the acute phase of ischaemic stroke from any cause and the risks of antiplatelet therapy in the presence of haemorrhagic infarction are uncertain, but the overall benefit in reducing the risk of vascular events by $25 \%$ in long term secondary prevention after stroke is clear. ${ }^{35}$ A small proportion of patients with stroke are already on anticoagulants or antiplatelet agents at the time of their stroke, and CT scanning is therefore needed to guide further treatment (stop if CT shows haemorrhage; continue if haemorrhage excluded). An additional need for CT occurs when a stroke patient develops a deep venous thrombosis (DVT). Up to $50 \%$ of paralysed legs had a DVT on fibrinogen scanning in one hospital series. ${ }^{36}$ If full-dose anticoagulation is planned in the treatment of established DVT or pulmonary embolism after stroke, CT scanning is required to exclude haemorrhage before treatment is started. It is not known whether it is safe to give low-dose subcutaneous heparin in the prophylaxis of DVT after stroke without first excluding intracranial haemorrhage. Finally, a small proportion of patients with minor ischaemic strokes in the carotid distribution may be suitable for carotid endarterectomy. Since minor haemorrhagic strokes are clinically indistinguishable from minor ischaemic strokes, patients with mild strokes should have early CT to avoid patients with haemorrhagic strokes being inappropriately put forward for angiography and carotid surgery.

\section{(3) Making a positive diagnosis of cerebral infarc-} tion Cerebral infarction may not be apparent in the first 24-48 hours, or the subtle area of hypodensity induced by early cellular oedema may be overlooked, and CT may not show an infarct in approximately $40 \%$ of cases. ${ }^{29}$ Over the following days increasing oedema may result in the appearance of a mass effect with displacement of normal tissue. Mass effect is greater with larger infarcts and is present to some degree in $70 \%$ of infarcts visible on CT in the first week. ${ }^{37}$ From $1-3$ weeks after stroke, infiltration of macrophages and other inflammatory cells involved in neuronal repair may cause the infarcted tissue to become isodense on CT scans, the 'fogging effect'. This effect occurs in approximately $50 \%$ of infarcts which were initially hypodense on early scans. ${ }^{38}$ One way to increase the positive diagnosis of cerebral infarction during this period is to repeat the scan with injection of iodinated contrast agents. This may show enhancement surrounding the area of infarction corresponding to neovascularization and increased permeability of the blood-brain barrier. Contrast scanning revealed significant enhancement in all patients whose infarcts had become 'fogged' at 2 weeks after ictus in one prospective series of 59 patients, ${ }^{39}$ but provided little additional information in those patients who had shown hypodense lesions on early scans. It has also been suggested in one retrospective study that the administration of contrast might adversely affect prognosis although the group given contrast also had an excess of several other markers of poor prognosis and this work needs confirmation. ${ }^{40}$ Late CT scans after several months may show hypodensity or localized atrophy corresponding to the area of infarction, but may also reveal no abnormality in up to $20 \%$ of cases despite a very clear clinicab picture of stroke. ${ }^{38}$ The overall accuracy of $C$ scanning in making a positive diagnosis of cerebrab infarction critically depends on the site and size of the infarct and the timing of the scan.

(4) Identifying unusual treatable forms of $\frac{\circ}{\triangleright}$ stroke Some uncommon forms of stroke will benefit from early diagnosis by CT scan, particularly cerebellar haemorrhage or infarction which presents with sudden headache, vertigo, and unsteadiness or slurred speech. In cases where cerebellar swelling results in obstructive hydrocephalus with worsening neurological deficit and declining conscious level CT scanning identifies the cases which may benefit from immediate neurosurgical intervention.

Cerebral vein thrombosis presents subacutely with headache, seizures, focal neurological deficit and altered conscious state. Early CT scan may show parasagittal low density areas suggesting venous infarction, particularly if there is associated haemorrhage or a filling defect in the superior sagittal sinus on axial slices with the injection of contrast (delta or empty triangle sign). ${ }^{41}$ Anticoagulant therapy, even in the presence of haemorrhage, has been shown to improve outcome in the largest series of these patients reported to date..$^{42}$

(5) Other indications Certain features on CT scanning such as the size of the area of infarction or haemorrhage, presence of mass effect, and site of (n)

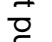

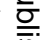


lesion correlate with functional outcome, ${ }^{43,44}$ but provide little additional information above that available from clinical features alone. Precise anatomical localization of infarcts is important for research purposes and may help if carotid endarterectomy is considered, but provides little help to the average stroke patient.

A summary of the indications for CT scanning in suspected stroke is provided in Table V. Data on the cost-effectiveness of CT scanning and its impact on management of stroke patients are scanty. ${ }^{45,46} \mathrm{~A}$ cost-effectiveness study in Sweden found that early CT scanning was more cost-effective than a policy of sequential investigation including CSF analysis and radionuclide scanning, with the detection of two non-stroke lesions on CT in 256 patients investigated which were missed with CSF and radionuclide scanning alone. ${ }^{47}$ Early CT might reduce the cost of hospital stay by earlier diagnosis of potentially disabling yet treatable conditions and by avoiding the occurrence of disabling cerebral haemorrhages in patients inappropriately treated with anticoagulants.

\section{(D) Diagnosis of cardiac sources of emboli and echocardiography}

Two-dimensional echocardiography is now widely available. This non-invasive investigation is relatively simple to perform and may identify a potential cardioembolic cause of ischaemic stroke. However, the application of this test to all patients with stroke would involve a huge commitment of resources from cardiology services (in the USA, the cost would be perhaps $\$ 100$ million dollars annually ${ }^{48}$ ).

Cardioembolic stroke is a clinical diagnosis made by excluding atherothrombotic disease in the artery supplying the area of the stroke, along with finding a cardiac lesion likely to cause emboli to the brain. Clinical features suggesting, but not diagnostic of, cardiogenic embolism include: ${ }^{49}$

(1) cerebral infarcts occurring in multiple vascular territories;

(2) peripheral emboli (such as femoral, mesenteric or renal arteries) in the presence of stroke;

(3) presence of cardiac thrombus on 2D echocardiography;

(4) presence of a potential embolic source (Table VI);

(5) absence of atherosclerotic disease in the symptomatic cerebral artery (usually diagnosed with non-invasive arterial imaging).

Pathological confirmation of cardiac embolism is rare and only in the minority of cases is a thrombus demonstrable in the heart by present techniques. Current echocardiographic techniques can detect a thrombus perhaps as small as
Table $\mathbf{V}$ Indications for CT scanning in patients with suspected stroke

1) Doubt about the diagnosis of stroke because no clear history is available (the patient is in coma, confused or dysphasic) or the presentation is atypical (gradual onset)

2) A clinical picture of cerebellar or 'brainstem' stroke, accompanied by declining conscious level raising the possibility of secondary obstructive hydrocephalus

3) Exclusion of intracranial haemorrhage in those patients already on antiplatelet or anticoagulant drugs, or in whom the use of such drugs, thrombolytic agents or carotid endarterectomy is planned

4) Atypical progression of the stroke after onset

5) Subarachnoid haemorrhage

6) Young patient without obvious risk factors for stroke

Table VI Cardiac abnormalities which are associated with an increased risk of ischaemic stroke (echocardiography probably cost effective)

HIGH RISK

Rheumatic mitral stenosis and atrial fibrillation

Mechanical prosthetic cardiac valves

Recent large anterior transmural myocardial infarction

Ventricular aneurysm with visible thrombus

Bacterial or marantic endocarditis

Cardiac myxoma

\section{LOWER RISK}

Mitral valve prolapse (with myxomatous degeneration)

Dilated cardiomyopathy and/or cardiac failure

Congenital heart disease with pulmonary hypertension

Calcific aortic or mitral valve disease

Atrial fibrillation without valvular heart disease

Sick sinus syndrome or left bundle branch block

Patent foramen ovale

Tissue heart valves

* In addition echocardiography may also be cost-effective in patients aged less than 45 years in whom no obvious cause of stroke can be found.

$5-10 \mathrm{~mm}$ in diameter, but a thrombus of only 1-2 mm diameter is sufficient to occlude a branch of the middle cerebral artery. Furthermore, echocardiography after stroke may be 'negative' if the thrombus which caused the stroke has left the heart and embolized to the brain! Standard 2D echocardiography does not visualize the atrial appendices well yet postmortem studies suggest that this is a common site of thrombus. The presence of a cardioembolic source does not necessarily prove that the stroke is due to cerebral infarction; in a Swedish series, $6 \%$ of patients with stroke who were in atrial fibrillation turned out, on 
CT scanning, to have a stroke due to primary intracerebral haemorrhage. ${ }^{50}$

Problems arise in the interpretation of the clinical picture, the definition of what is a potential source of embolism, and definition of what represents atherosclerotic disease. A reasonable consensus seems to be that somewhere between $6-23 \%$ of strokes may be caused by cardiac emboli. ${ }^{51}$ It has been thought worthwhile to make this diagnosis because a cardiac source of embolus carries a high risk of recurrence (possibly in a vascular territory remote from the initial insult), and many clinicians use urgent anticoagulation in this situation. This is probably reasonable in patients at very high risk of recurrence (mechanical prosthetic valves, rheumatic mitral stenosis with atrial fibrillation, left ventricular thrombi), but in other groups at lower risk (non-valvular atrial fibrillation, tissue valves, mitral valve prolapse) the role of anticoagulation is unclear and is currently being tested in trials such as the European Atrial Fibrillation Trial. There is a $\mathbf{4 0 \%}$ risk of haemorrhagic transformation in cardioembolic cerebral infarcts, ${ }^{33}$ data from current randomized trials will provide clearer evidence on whether the presence of haemorrhagic transformation should be an absolute or relative contraindication to the use of anticoagulants.

Echocardiography represents a simple noninvasive way of identifying potential cardiac sources of emboli. The yield of echocardiographic abnormalities is much greater in patients who have evidence of cardiac abnormality on clinical history and examination (supported by chest $\mathrm{X}$-ray and ECG). In several studies, detailed echocardiography identified abnormalities associated with cardiogenic embolism in $6-30 \%$ of cases with clinical evidence of cardiac disease and only $0-3 \%$ of patients with no clinical evidence of cardiac disease. ${ }^{52-56}$ In a retrospective study Robbins $e t$ $a l .{ }^{56}$ calculated that it cost approximately $\$ 1,200$ US (1983 prices) for every high-risk lesion identified in patients with known cardiovascular disease, and that $30 \%$ of these patients had their treatment changed as a result of $2 \mathrm{D}$ echocardiography. Groups in which echocardiography would seem cost-effective in the diagnosis of potential cardiac sources of embolism are listed in Table VI.

Transoesophageal echocardiography is a new technique currently being evaluated. An oesophageal probe is used to visualize the left ventricle, atria, mitral valve apparatus, aortic valve, interatrial septa and aortic root. It is an ideal technique for evaluating the atrial appendices. Recent studies suggest that transoesophageal echocardiography has a much greater sensitivity than praecordial echocardiography, although the ventricular apex is usually not well seen using the former tech- nique. ${ }^{57-59}$ The transoesophageal approach is timeconsuming and carries a small risk, since it requires? local anaesthesia to the pharynx and sometimes $\stackrel{\mathbb{Q}}{\stackrel{1}{2}}$ sedation in patients who may be restless. It mayc. become necessary in some patients to perform both $\vec{\Rightarrow}$ praecordial and transoesophageal techniques, $\stackrel{?}{?}$ greatly increasing time required and cost involved.

\section{(E) Cerebral angiography in patients with stroke}

Moniz first diagnosed occlusive cerebrovascular disease in 1937 using the technique of carotid $\vec{P}$ angiography in four patients with episodes of ${ }^{\circ}$ weakness. ${ }^{60}$ Present indications for angiography in $\vec{\omega}$ the acute phase of ischaemic stroke are limited to $\frac{\rho}{8}$ the diagnosis of non-atherogenic cerebral vesseko occlusion such as that caused by carotid artery dissection, Moya Moya disease or vasculitis, or $\sqrt{ }$ ischaemic stroke in a young patient with no obvious causative factors.

In the future, some assessment of the patency of $\sim$ intra- and extra-cranial arteries may be required before treatment with thrombolytic therapy in 을 acute ischaemic stroke. In a recent series Fieschi et $\overrightarrow{-}$ al..$^{34}$ demonstrated complete occlusion of the apropriate cerebral artery in $76 \%$ of patients with $\stackrel{\frac{\pi}{9}}{3}$ acute ischaemic stroke who underwent angiography within 6 hours of stroke onset. Sugig 8 patients may be suitable for entry in randomized. trials of thrombolytic therapy, although patiers who might have most to gain would be those with $\frac{\mathrm{a}}{\mathrm{a}}$ viable ischaemic 'penumbra' distal to the site of $\frac{\partial}{0}$ vascular occlusion. The assessment of the site and

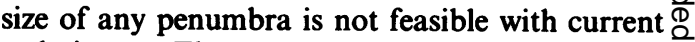
techniques. There were 2 cases $(2.5 \%, 95 \% \stackrel{2}{\vec{F}}$ confidence interval $0.3-8.8 \%$ ) of clinical deteriora- $\frac{0}{3}$ tion following angiography in this series, ${ }^{34}$ angiography in the acute phase therefore carries a significant risk.

Angiography may be indicated in patients who have made a good recovery from a mild ischaemic $\dot{\sigma}$. stroke, if carotid endarterectomy to prevent further stroke is being considered. In this situation, the risk $\delta$ of permanent neurological deficit as a complication $₹$ of angiography is approximately $1 \% .{ }^{61}$ This comp- 0 lication rate should be added to the $3-4 \%$ risk of disabling stroke/death associated with carotid o surgery. Intravenous digital subtraction angiography fails to provide the anatomical definition os required to plan carotid surgery and carries a $17 \%$ N risk of systemic complications associated with the $\mathrm{N}_{\mathrm{N}}$ administration of large volumes of contrast 0 material. ${ }^{62}$ As a screening test to select patients for intra-arterial angiography, duplex ultrasound $\underset{\Phi}{\bar{D}}$ examination of the carotid extracranial circulation $\stackrel{\oplus}{\rightarrow}$ offers a sensitivity and specificity comparable to 0 that of intravenous digital subtraction angio- 0 graphy without the potential complications. ${ }^{63}$ 
New investigations limited to research at present but which may be used in routine clinical application in the future

\section{(A) Transcranial Doppler ultrasonography (TCD)}

This technique uses a low-frequency pulsed ultrasound beam and a detector which can record flow velocities at selected distances from the probe. The beam can be directed through an 'orbital' window to record information about the carotid siphon, a 'temporal' window to record velocities in the middle, posterior and anterior cerebral arteries, and the foramen magnum to record velocities in the vertebrobasilar system. Absolute velocity measurements do not correlate well with cerebral blood flow for a number of reasons but comparison of velocities with the contralateral hemisphere and assessment of collateral and retrograde flow gives information about proximal occlusion of the major cerebral vessels. In $5-15 \%$ of patients it is not possible to make satisfactory recordings through the temporal window as a result of the thickness of the vault or anatomical variations in the blood vessels. ${ }^{64}$ Each examination can take a long time (up to 1 hour per examination) and the diagnostic accuracy is highly dependent on the experience of the operator. In future it may provide an alternative to angiography in the early diagnosis and follow-up of intracranial arterial occlusion in acute ischaemic stroke. Preliminary studies suggest a high sensitivity and specificity for the diagnosis of proximal middle cerebral artery occlusion. ${ }^{65}$

\section{(B) Nuclear magnetic resonance imaging ( NMR)}

This non-invasive technique records the highfrequency radio waves emitted by protons as they are manipulated in a magnetic field. Measures of proton density and relaxation curves allow discrimination between different tissue types. T1weighted images reflect differences in the rate of tissue magnetization and T2-weighted images reflect differences in the rate of signal decay between different tissue types. Early cerebral infarcts (within hours of onset) appear as low signal regions on $\mathrm{Tl}$-weighted images and as regions of increased signal with T2- weighting. NMR detects areas of infarction, including small lacunar lesions, earlier and with greater sensitivity than CT scanning, ${ }^{66-69}$ and may be especially helpful in the posterior fossa where conventional CT scans are often degraded by bony artifact. ${ }^{70} \mathrm{CT}$ scanning remains the method of choice in the detection of cerebral haemorrhage, as NMR scans performed less than 24 hours after the onset may fail to reveal the haemorrhage. ${ }^{69}$ Enhancement of cerebral infarcts on NMR scans occurs with the use of gadolinium contrast during the period of neovascularization and blood-brain barrier breakdown in the 1-3 weeks after stroke onset.

A new application of NMR scanning in acute stroke is spectroscopy which enables measurement of ATP, lactate levels and $\mathrm{pH}$ at discrete locations within the brain. This may help identify potentially viable infarct tissue which could benefit from revascularization. ${ }^{71}$ Another NMR technique currently being evaluated is angiography with digital reconstruction of arterial flow patterns. Current techniques are a long way from providing pictures superior to conventional arteriography, ${ }^{72,73}$ although the potential advantages of a technique such as this, which is non-invasive and requires no contrast agent, are obvious. NMR scanning takes quite a long time to perform (30-45 minutes) and some patients find the scanner itself claustrophobic, both factors which reduce utility in the unwell acute stroke patient who may be also restless.

\section{(C) Single photon emission computed tomography (SPECT)}

This radioisotope technique measures cerebral blood flow, and, when combined with a tomographic scanner, allows two-dimensional reconstruction of cerebral blood flow patterns. These patterns are a functional representation of the cerebral infarct and correlate well with clinical signs and subsequent CT images. Tracers such as ${ }^{123}$ I iodoamphetamine or ${ }^{99 \mathrm{~m}}$ technetium HMPAO give a qualitative picture of cerebral bloodflow in the infarct area. Changes in response to vasodilators such as acetazolamide assess the integrity of cerebral autoregulation and may give some idea of tissue viability. ${ }^{74}$ SPECT studies may be more sensitive than CT scanning in the detection of early cerebral infarction but cannot reliably diagnose haemorrhage or predict prognosis. ${ }^{75,76}$ ${ }^{133}$ Xenon contrast CT scans are an alternative method of measuring cerebral blood flow but require the patient to remain immobile for 15-20 minutes whilst the scan is performed, and xenon has soporific effects and may alter flow by acting as a vasodilator. Xenon provides a quantitative measure of cerebral blood flow correlating well with other methods. ${ }^{77}$

\section{(D) Positron emission tomography (PET)}

Certain radio-isotopes decay spontaneously, emitting a small positively charged particle (positron), which interacts with an electron, producing gamma radiation which is detectable with a gamma camera. This technique enables precise and quantitative analysis of radioisotope concentrations from 
molecules such as ${ }^{11} \mathrm{C},{ }^{13} \mathrm{~N},{ }^{15} \mathrm{O}$ and ${ }^{18} \mathrm{~F}$ which may be attached to normal metabolic substances such as oxygen, glucose and water. Unfortunately, these radio-isotopes can only be produced using a cyclotron and have short half-lives (between a few minutes and two hours) which restricts their use to a few centres in the world. Quantitative regional measurements can be made of cerebral blood flow, blood volume, oxygen uptake and glucose metabolism enabling definition of normal brain from infarct, and the region of ischaemic but potentially viable brain surrounding the infarct (the 'ischaemic penumbra'). ${ }^{78}$ Areas of altered metabolism distant to the area of infarction have also been identified which may have an important pathophysiological role (diaschisis). Metabolic abnormalities on PET scanning may predict the development of future structural lesions and act as a guide to appropriate treatment in acute cerebral infarction, although its potential for widespread use is limited because of the enormous costs involved.

\section{Conclusion}

Many investigations are now available for the assessment of patients with acute stroke. The

\section{References}

1. Malmgren, R., Bamford, J., Warlow, C., Sandercock, P. \& Slattery, J. Projecting the number of patients with first ever strokes and patients newly-handicapped by stroke in England and Wales. Br Med J 1989, 298: 656-660.

2. Isard, P.A. \& Forbes, J. Stroke and health service resource use in Scotland. Personal Communication.

3. Sandercock, P.A.G., Warlow, C.P., Jones, L.N. \& Starkey, I.R. Predisposing factors for cerebral infarction: the Oxfordshire community stroke project. $\mathrm{Br} \mathrm{Med} J \mathrm{1989}$, 298: 75-80.

4. Vermeulen, M. \& VanGijn, J. The diagnosis of subarachnoid haemorrhage. J Neurol Neurosurg Psychiatry 1990, 53: $365-372$.

5. Norris, J.W. \& Hachinski, V.C. Misdiagnosis of stroke. Lancet 1982 , i: $328-331$.

6. Isaacs, B. Five years' experience of a stroke unit. Health Bulletin 1977, 35: 94-98.

7. Allen, C.M.C. Clinical diagnosis of the acute stroke syndrome. $Q J$ Med 1983, 52: 515-523.

8. Barer, D.H. \& Mitchell, J.R. Predicting the outcome of acute stroke: do multivariate models help? $Q J$ Med 1989, 70: 27-39.

9. Jongbloed, L. Prediction of function after stroke. Stroke 1986, 17: 765-775.

10. Wade, D.T., Wood, V.A. \& Hewer, R.L. Recovery after stroke: the first three months. J Neurol Neurosurg Psychiatry 1985, 48: 7-13.

11. Prescott, R.J., Garraway, W.M. \& Akhtar, A.J. Predicting functional outcome following acute stroke using a standard clinical examination. Stroke 1982, 13: 641-647.

12. Fullerton, K.J., MacKenzie, G. \& Stout, R.W. Prognostic indices in stroke. $Q J$ Med 1988, 66: 147-162.

13. Sackett, D.L. How to read clinical journals: II. To learn about a diagnostic test. Can Med Assoc J 1981, 124: 703-710. investigations which need to be performed in anf individual patient will depend on patient charac $-\frac{2}{3}$ teristics, management priorities and local facilities $\mathbb{Q}$ A good clinical history and examination is the mostcost-effective investigation of all. The basic investi gations outlined in the first section are a cost effective addition to the clinical examination an $\bar{E}$ are applicable to virtually all stroke patients. $\mathrm{CT}$ 흘. scanning may be required in a large proportion of patients, the exact proportion depending largely on whether clinicians plan to use anti-haemostatiç agents for either acute treatment or long-termsecondary prevention, whereas other investigations such as echocardiography are indicated only in $\vec{\omega}$ selected patients in whom there is diagnostic uncer 2 tainty, or in whom management may be signie ficantly altered. The newer research techniques. outlined require further evaluation and mays assume great importance in the future when more effective therapies for the acute treatment of stroker are available.
14. Merritt, H.H. \& Sciarra, D. Disorders of the blood vessels of the brain and spinal cord. In: Harrison, T.R., Beeson, P.B., Resnik, W.H., Thorn, G.W., Wintrobe, M.M. (eds) Principles of Internal Medicine. Blakiston, Philadelphia 1950, $\overrightarrow{\vec{\sigma}}$ pp. 1514-1520.

15. Kushner, M., Nencini, P., Reivich, M. et al. Relation of hyperglycaemia early in ischaemic brain infarction to cere bral anatomy, metabolism, and clinical outcome. Ann Neurol 1990, 28: 129-135.

16. Williams, K. Screening for syphilis in pregnancy: an assess 3 . ment of the costs and benefits. Commun Med 1985, 7: 37-42.

17. Norris, J.W. \& Hachinski, V.C. Stroke units or stroke centers? Stroke 1986, 17: 360-362.

18. Levine, S.R. \& Welch, K.M.A. Antiphospholipid antibodies. Ann Neurol 1989, 26: 386-389.

19. Landi, G., D’Angelo, A., Boccardi, E. et al. Hyper-은 coagulability in acute stroke: prognostic significance. Neurology 1987, 37: 1667-1671.

20. Britton, M., Hultman, E., Murray, V. \& Sjoholm, H. The diagnostic accuracy of CSF analyses in stroke. Acta Med
Scand 1983, 214: 3-13.

21. Ruff, R.L. \& Dougherty, J.H. Evaluation of acute cerebralN ischaemia for anticoagulant therapy: computed tomography or lumbar puncture. Neurology 1981, 31: 736-740.

22. Bories, J., Derhy, S. \& Chiras, J. CT in hemispheric ischaemic이 attacks. Neuroradiology 1985, 27: 468-483.

23. Shinar, D., Gross, C.R., Hier, D.B. et al. Interobserver reliability in the interpretation of computed tomographic scans of stroke patients. Arch Neurol 1987, 44: 149-155.

24. Sandercock, P.A.G., Molyneux, A. \& Warlow, C. Value of $\square$ computed tomography in patients with stroke: Oxfordshire $\bar{O}$ Community Stroke Project. Br Med J 1985, 290: 193-197. 
25. Hewer, R. \& Wood, V.A. Availability of computed tomography of the brain in the United Kingdom. Br Med J 1989, 298: $1219-1220$.

26. Matenga, J., Kitai, I. \& Levy, L. Strokes among black people in Harare, Zimbabwe: results of computed tomography and associated risk factors. $\mathrm{Br}$ Med J 1986, 292: 1649-1651.

27. Britton, M., Hindmarsh, T., Murray, V. \& Tyden, S.A Diagnostic errors discovered by CT in patients with suspected stroke. Neurology 1984, 34: 1504-1507.

28. Weisberg, L.A. \& Nice, C.N. Intracranial tumours simulating the presence of cerebrovascular syndromes: early detection with cerebral computed tomography. Am J Med 1977, 63: 517-524.

29. Campbell, J.K., Houser, O.W., Stevens, J.C., Wahner, H.W., Baker, H.L. \& Folgar, W.N. Computed tomography and radionuclide imaging in the evaluation of ischaemic stroke. Radiology 1978, 126: 695-702.

30. Kinkel, W.R. \& Jacobs, L. Computerized axial transverse tomography in cerbrovascular disease. Neurology 1976, 26: 924-930.

31. Weisberg, L.A. Computerized tomography in intracranial haemorrhage. Arch Neurol 1979, 36: 422-426.

32. Dennis, M.S., Bamford, J.M., Molyneux, A.J. \& Warlow, C.P. Rapid resolution of signs of primary intracerebral haemorrhage in computed tomograms of the brain. $\mathrm{Br} \mathrm{Med} \mathrm{J}$ 1987, 295: 379-381.

33. Okada, Y., Yamaguchi, T., Minematsu, K. et al. Haemorhagic transformation in cerebral embolism. Stroke 1989, 20: 598-603.

34. Fieschi, C., Argentino, C., Lenzi, G.L., Sacchetti, M.L., Toni, D. \& Bozzao, L. Clinical and instrumental evaluation of patients with ischaemic stroke within the first six hours. $J$ Neurol Sci 1989, 91: 311-322.

35. Antiplatelet Trialists' Collaboration. Secondary prevention of stroke, myocardial infarction and vascular death by prolonged antiplatelet treatment. $J$ Neurol 1990, 237: 130.

36. Warlow, C., Ogston, D. \& Douglas, A.S. Deep venous thrombosis of the legs after strokes. Part 1 - Incidence and predisposing factors. $\mathrm{Br}$ Med J 1976, 1: 1178-1183.

37. Masdeu, J.C., Azar-Kia, B. \& Rubino, F.A. Evaluation of recent cerebral infarction by computed tomography. Arch Neurol 1977, 34: 417-421.

38. Skriver, E.B. \& Olsen, T.S. Transient disappearance of cerebral infarcts on CT scan, the so-called fogging effect. Neuroradiology 1981, 22: 61-65.

39. Skriver, E.B. \& Olsen, T.S. Contrast enhancement of cerebral infarcts: incidence and clinical value in different states of cerebral infarction. Neuroradiology 1982, 23: 259-265.

40. Kendall, B.E. \& Pullicino, P. Intravascular contrast injection in ischaemic lesions. II. Effect on prognosis. Neuroradiology 1980, 19: $241-243$.

41. Banerjee, A.K. \& Carvalho, P. An unusual cause for a fit. Postgrad Med J 1990, 66: 842-843.

42. Einhaupl, K.M., Villringer, A., Haberl, R.L., Dirnagl, U.D., Pfister, H.W. \& Schmiedek, P. Diagnosis of sinus venous thrombosis. J Neurol 1990, 237: 159.

43. Valdimarsson, E., Bergvall, U. \& Samuelsson, K. Prognostic significance of cerebral computed tomography results in supratentorial infarction. Acta Neurol Scand 1982, 65: $133-145$.

44. Brott, T. \& Reed, R.L. Intensive care for acute stroke in the community hospital setting: the first 24 hours. Stroke 1989, 20: 694-697.

45. Hazelton, A.E. \& Earnest, M.P. Impact of computed tomography on stroke management and outcome. Arch Intern Med 1987, 147: 217-220.

46. Larson, E.B., Omenn, G.S. \& Loop, J.W. Computed tomography in patients with cerebrovascular disease: impact of a new technology on patient care. Am J Roentgenol 1978, 131: $35-40$.

47. Britton, M., Jonsson, E., Marke, L.A. \& Murray, V. Diagnosing suspected stroke: a cost-effectiveness analysis. Int $J$ Tech Assess 1985. 1: 147-158.
48. Larson, E.B., Stratton, J.R. \& Pearlman, A.S. Selective use of two-dimensional echocardiography in stroke syndromes. Ann Intern Med 1981, 94: 112-113.

49. Cerebral Embolism Task Force. Cardiogenic brain embolism. Arch Neurol 1986, 43: 72-81.

50. Britton, M. \& Gustafsson, C. Non-rheumatic atrial fibrillation as a risk factor for stroke. Stroke 1985, 16: 182-187.

51. Cerebral Embolism Task Force. Cardiogenic brain embolism: the second report of the cerebral embolism task force. Arch Neurol 1989, 46: 727-743.

52. Greenland, P., Knopman, D.S., Mikell, F.L., Asinger, R.W., Anderson, D.C. \& Good, D.C. Echocardiography in diagnostic assessment of stroke. Ann Intern Med 1981, 95: 51-53.

53. Knopman, D.S., Anderson, D.C., Asinger, R.W., Greenland, P., Mikell, F. \& Good, D.C. Indications for echocardiography in patients with ischaemic stroke. Neurology 1982, 32: 1005-1011.

54. Burnett, P.J., Greenwood, R., Milne, J.R., Giles, M.R. \& Camm, J. The role of echocardiography in the investigation of focal cerebral ischaemia. Postgrad Med J 1984, 60: $116-119$.

55. Lovett, J.L., Sandok, B.A., Giuliani, E.R. \& Nasser, F.N. Two-dimensional echocardiography in patients with focal cerebral ischaemia. Ann Intern Med 1981, 95: 1-4.

56. Robbins, J.A., Sagar, K.B., French, M. \& Smith, P.J. Influence of echocardiography on management of patients with systemic emboli. Stroke 1983, 14: 546-549.

57. Voll, C.L., Polasek, P., Cujec, B. \& Shuaib, A. Transoesophageal echocardiography improves diagnosis of potential cardioembolic sources in unselected stroke patients. Can J Neurol Sci 1990,17:229-Ab 66.

58. Pop, G., Sutherland, G.R., Koudstaal, P.J., Sit, T.W., De Jong, G. \& Roelandt, R.T.C. Transoesophageal echocardiography in the detection of intracardiac embolic sources in patients with TIAs. Stroke 1990, 21: 560-565.

59. Stollberger, C., Brainin, M., Slany, J. \& Wien, R. Detection of paradoxical embolism by combined use of transoesophageal echocardiography (TEE) and phlebography. J Neurol 1990, 237: 144.

60. Gurdjian, E.S. History of occlusive cerebrovascular disease: II After Moniz, with special reference to surgical treatment. Arch Neurol 1979, 36: 427-432.

61. Hankey, G.J., Warlow, C.P. \& Sellar, R.J. Cerebral angiographic risk in mild cerebrovascular disease. Stroke 1990, 21: 209-222.

62. Cebul, R.D. \& Paulus, R.A. The failure of intravenous digital subtraction angiography in replacing carotid arteriography. Ann Intern Med 1986, 104: 572-574.

63. Tans, J.T.J. \& Hoogland, P.H. The role of digital subtraction angiography of the carotid bifurcation in the evaluation of patients with reversible ischaemic attacks or stroke. Stroke 1985, 16: 435-440.

64. Caplan, L.R., Brass, L.M., DeWitt, L.D. et al. Transcranial Doppler ultrasound: present status. Neurology 1990, 40: 696-700.

65. Zanette, E.M., Fieschi, C., Bozzao, L. et al. Comparison of cerebral angiography and transcranial Doppler sonography in acute stroke. Stroke 1989, 20: 899-903.

66. Hommel, M., Besson, G., Le Bas, J.F. et al. Prospective study of lacunar infarction using magnetic resonance imaging. Stroke 1990, 21: 546-554.

67. Brant-Zawadzki, M., Weinstein, P., Bartkowski, H. \& Moseley, M. MR imaging and spectroscopy in clinical and experimental cerebral ischaemia: a review. Am $J$ Roentgenol 1987, 148: $579-588$.

68. Ramadan, N.M., Deveshwar, R. \& Levine, S.R. Magnetic resonance and clinical cerebrovascular disease: an update. Stroke 1989, 20: 1279-1283.

69. Kertesz, A., Black, S.E., Nicholson, L. \& Carr, T. The sensitivity and specificity of MRI in stroke. Neurology 1987 , 37: $1580-1585$. 
70. Davis, S.M., Tress, B.M., Dowling, R., Donnan, G.A., Kiers, L. \& Rossiter, S.C. Magnetic resonance imaging in posterior circulation infarction: impact on diagnosis and management. Aust NZ J Med 1989, 19: 219-225.

71. Felber, S.R., Aichner, F.T. \& Sauter, R. Sequential 1H MR-spectroscopy: new insights into ischaemic mechanisms. J Neurol 1990, 237: 156-157.

72. Haacke, E.M. \& Masaryk, T.J. The salient features of MR angiography. Radiology 1989, 173: 611-612.

73. Ross, J.S., Masaryk, T.J., Modic, M.T., Harik, S.I., Wiznitzer, M. \& Selman, W.R. Magnetic resonance angiography of the extracranial carotid arteries and intracranial vessels: a review. Neurology 1989, 39: 1369-1376.

74. Raynaud, C., Rancurel, G., Tzourio, N. et al. SPECT analysis of recent cerebral infarction. Stroke 1989, 20: 192-204.
75. Hayman, L.A., Taber, K.H., Jhingran, S.G., Killian, J.M. \& Carroll, R.G. Cerebral infarction: diagnosis and assessment of prognosis by using 123IMP-SPECT and CT. AJNR 1989 10: $557-562$.

76. Fazekas, F., Valetitsch, H., Schmidt, R. et al. Imaging considerations for the early detection of cerebral ischaemic lesions. J Neurol 1990, 237 : 147.

77. Hughes, R.L., Yonas, H., Gur, D. \& Latchaw, R. CerebraP blood flow determination within the first 8 hours of cerebra infarction using stable xenon-enhanced computed tomo $\overline{\bar{w}}$. graphy. Stroke 1989, 20: 754-760.

78. Powers, W.J.\& Raichle, M.E. Positron emission tomograph $\mathbb{\varnothing}$ and its application to the study of cerebrovascular disease in man. Stroke 1985, 16: 361-376. 